\title{
Analysis of discontinuities in waveguiding structures by MAB method
}

\author{
J. Macháč
}

Indexing terms: Microstrip, Waveguides, Electromagnetics

\begin{abstract}
The method of minimal autonomous blocks is briefly described. A universal code computing reflectivity and transmissivity of discontinuities in waveguide structures was developed on the basis of the MAB method. This technique is applied to the solution of dielectric cylinders, metal ridges and bandpass filters in a rectangular waveguide, as well as on shunt posts in a microstrip line. Special attention was given to E-plane tee junctions in rectangular waveguides; scattering matrices are evaluated and approximated by the proper function with dependence on frequency and branch waveguide height.
\end{abstract}

\section{Introduction}

In many cases one needs to determine the characteristics of various structures used in microwave and millimetrewave techniques. A number of evaluation methods have been published in the literature, some of which are mentioned in the text. For every structure the standard, more or less optimal method is usually applied; other structures require a new technique. So it is useful to have some universal tool which is applicable to a wide class of problems yielding results to at least a first approximation. The aim of this work is to report on such approach to various structures analysis, to show its flexibility and the broad range of possible applications that could be solved using the technique described.

A universal code has been developed, first reported by the author in Reference 1 . It evaluates the reflectivity and transmissivity of an arbitrary obstacle or an inhomogeneity in a rectangular waveguide. There are some other versions of this code that enable solution of microstrip lines, tee junctions and to calculate propagation constants and transverse distribution of modes in waveguides having a complex cross-section. The code is based on the method of minimal autonomous blocks (MAB) worked out originally in Reference 2 . This method uses the decomposition of an analysed region to partial prisms (blocks) small enough so that the electromagnetic field could be assumed to be constant on their faces. These blocks are described by scattering matrices which are combined in a resulting matrix containing the required results.

The MAB method and the code are briefly described. The results of $2 \mathrm{D}$ and $3 \mathrm{D}$ modelling of inhomogeneities

Paper 8835H (E12), first received 28th October 1991 and in revised form 12th March 1992

The author is with the Faculty of Electrical Engineering, Czech Technical University, Technická 2, 16627 Prague 6, Czechoslovakia in a rectangular waveguide are shown. Bandpass filters with the shunt inductive obstacles are analysed. Further, the code is applied to the solution of microstrips in $2 \mathrm{D}$ using a homogeneous equivalent waveguide and to a shielded microstrip in 3D. The detailed solution of the E-plane tee junction in a rectangular waveguide was performed. Dependence on frequency and branch waveguide height of the scattering matrix of this three-port junction is a result.

All the values obtained are compared either with measured data or with data published in the literature.

\section{Theoretical background and code description}

This section reviews the substance of the MAB method with the main aim of evaluating the reflectivity and transmissivity of an obstacle in a rectangular waveguide for the dominant mode of propagation.

The region of a waveguide with an analysed obstacle is extended by the neighbouring parts that ensure decay of excited higher modes. So only the dominant mode on the input and output planes (ports) is taken into account. The whole volume is divided by a system of planes to the prisms denoted minimal autonomous blocks (MAB) see Fig. 1A. These blocks must be small enough to assume constant electromagnetic field on their faces. It should be written as a superposition of two partial waves

$$
E_{t}=\sum_{n=1}^{2} a_{n} e_{n} \quad H_{t}=\sum_{n=1}^{2} b_{n} h_{n}
$$

Each face is assumed as a virtual waveguide with eigenwaves $\boldsymbol{e}_{n}, \boldsymbol{h}_{n}$. Such blocks are characterised by the scattering matrices of minimal order, 12 for $3 D$ and 4 for $2 D$ (that is why the blocks are denoted as minimal). Solving the diffraction of the partial electromagnetic waves (eqn. 1) independently in each block their scattering matrices can be determined (hence the notation 'autonomous').

The precise solution of Maxwell's equations (involving all vector components) under the incidence of a wave with unit amplitude on the face $m$ allows determination of the field inside the block. Complex amplitudes $c_{n}^{-}$of waves outgoing from each block face $n$ are evaluated as coefficients of generalised Fourier series of tangential field on the face $n$ :

$$
\left(\begin{array}{l}
E_{n t} \\
H_{n t}
\end{array}\right)=\sum_{k=-\infty}^{\infty} c_{n k}\left(\begin{array}{l}
\boldsymbol{e}_{n k} \\
\boldsymbol{h}_{n k}
\end{array}\right)
$$

The solution in 3D was performed on an IBM 3090 computer, with acknowledgement to the IBM Academic Initiative in Czechoslovakia. 
For outgoing waves $(k<0)$ is $c_{n k}=c_{n|k|}^{-}$. In the case of a minimal block eqn. 2 reduces to eqn. 1 and there are only four nonzero coefficients $c_{n k}$ and $c_{n k}^{-}$where $k= \pm 1, \pm 2$. The amplitudes $c_{n k}^{-}$are equal to the adequate $S$-matrix elements $S_{n m}$.

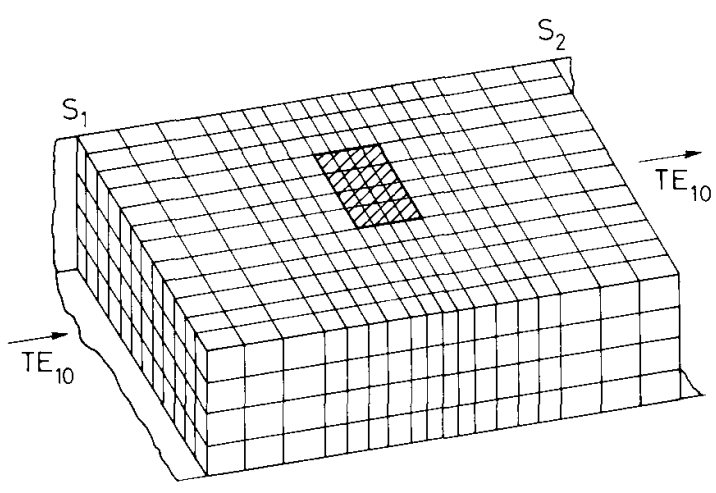

Fig. 1A Analysed rolume and its discretisation to minimal autonomous blocks

$S_{1}$ and $S_{2}$ are input and output planes, respectively

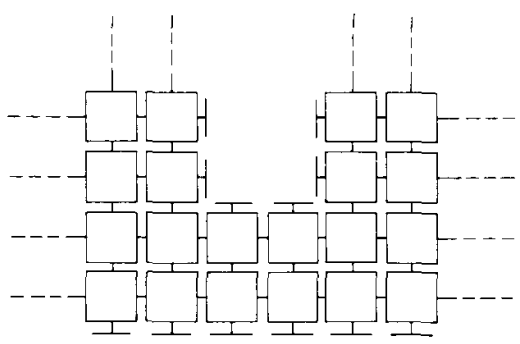

Fig. 18 Part of equivalent electrical network

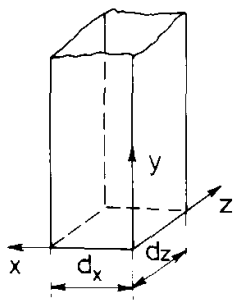

Fig. $1 \mathrm{C} 2 \mathrm{D}$ block

As an example, consider the scattering matrix of a block in 2D (in Fig. 1C). Solutions for partial waves in eqn. 1 can be separated and treated independently. In the case of the vector $\boldsymbol{E}$ parallel with the y-axis (shorter waveguide wall), eqn. 2 series has only two elements with $k= \pm 1$. The base vectors on the block walls are (co-ordinate system is introduced in Fig. 1C)

$$
\begin{array}{ll}
e_{ \pm 1}=y_{0} & e_{ \pm 2}=y_{0} \\
h_{ \pm 1}=\mp x_{0} / Z & h_{ \pm 2}= \pm x_{0} / Z \\
e_{ \pm 3}=y_{0} & e_{ \pm 4}=y_{0} \\
h_{ \pm 3}= \pm z_{0} / Z & h_{ \pm 4}=\mp z_{0} / Z
\end{array}
$$

352 where $Z$ is the characteristic impedance of material in the block. The scattering matrix has the form

$$
[S]=\left[\begin{array}{llll}
a_{1} & c_{1} & b_{2} & b_{2} \\
c_{1} & a_{1} & b_{2} & b_{2} \\
b_{1} & b_{1} & a_{2} & c_{2} \\
b_{1} & b_{1} & c_{2} & a_{2}
\end{array}\right]
$$

where

$$
\begin{aligned}
& a_{1}=j \frac{J_{z} J_{x}}{\sqrt{ }\left(T_{z}\right)\left[J_{z} J_{x}-\sqrt{\left.\left(T_{z} T_{x}\right)\right]}\right.} \sin \frac{k d_{z}}{2} \\
& b_{1}=-j \frac{J_{z}}{J_{z} J_{x}-\sqrt{ }\left(T_{z} T_{x}\right)} \sin \frac{k d_{x}}{2} \\
& c_{1}=\frac{1}{T_{z}}+a_{1} \\
& T_{x}=e^{j k d_{x}} \quad T_{z}=e^{j k d_{z}} \\
& J_{x}=\frac{2}{k d_{x}} \sin \frac{k d_{x}}{2} \quad J_{z}=\frac{2}{k d_{z}} \sin \frac{k d_{z}}{2}
\end{aligned}
$$

$k$ is the wave number, $d_{x}, d_{z}$ are block dimensions. Values $a_{2}, b_{2}, c_{2}$ are obtained from $a_{1}, b_{1}, c_{1}$, changing $x$ by $z$ in eqn. 5 and vice versa.

In 3D the solutions cannot be separated as in 2D because the partial waves are bound by the $S$-matrix that has an order equal to 12 . Examples of scattering matrices for various blocks are in Reference 2 .

Blocks form an electrical equivalent network which is partially shown in Fig. 1B. Each connection between two adjoining blocks is in fact created by two lines because there are two independent waves on each block face.

Conditions on a boundary of two materials with dif ferent parameters $\varepsilon, \mu, \sigma$ are satisfied by inclusion of a special auxiliary block ensuring the proper reflectivity and transmissivity in the virtual waveguide between two adjoining blocks. The $S$-matrix can be written

$$
\begin{aligned}
{[S]^{3 \mathrm{D}} } & =\left[\begin{array}{cccc}
R & 0 & T_{1} & 0 \\
0 & R & 0 & T_{1} \\
T_{2} & 0 & -R & 0 \\
0 & T_{2} & 0 & -R
\end{array}\right] \\
{[S]^{2 \mathrm{D}} } & =\left[\begin{array}{cc}
R & T_{1} \\
T_{2} & -R
\end{array}\right]
\end{aligned}
$$

where $R, T_{1}, T_{2}$ are reflectivity and transmissivities in both directions of the boundary, respectively. The $S$ matrix of the block filled by a well conducting material has the form of eqn. 6 with $T_{1}=T_{2}=0$.

Blocks ensuring boundary conditions on waveguide walls have the $S$-matrix

$$
[S]^{3 \mathrm{D}}=\left[\begin{array}{ll}
R & 0 \\
0 & R
\end{array}\right] \quad[S]^{2 \mathrm{D}}=R
$$

For the electric wall $R=-1$ and for the magnetic wall $R=1$.

The scattering matrix $\boldsymbol{S}_{\boldsymbol{c}}$ of the whole analysed volume could be obtained using methods well known from the theory of linear $n$-ports (combining blocks in the equivalent network). The order of this matrix is equal to the number of blocks in one transverse layer multiplied by four, which is the number of all partial waves on both planes $S_{1}$ and $S_{2}$.

Division of the analysed volume to blocks (see Fig. 1A) depends on its complexity. Blocks follow inner and outer

IEE PROCEEDINGS-H, Vol. 139, No. 4, AUGUST 1992 
boundaries. Curved boundaries must be approximated by steps (Fig. 3). The density of blocks (their dimensions) is determined by the speed of field changes; field on block faces should be constant. There is no universal rule for the creation of a block net.

Reflectivity $R$ and transmissivity $T$ of the dominant mode propagating in a waveguide are derived from the matrix $S_{c}$. This could be rewritten in the form

$$
\left[\begin{array}{c}
C_{1}^{-} \\
\hdashline C_{2}^{-}
\end{array}\right]=\left[\begin{array}{l:l}
S^{11} & S^{12} \\
\hdashline S^{21} & S^{22}
\end{array}\right]\left[\begin{array}{c}
C_{1}^{+} \\
C_{2}^{+}
\end{array}\right]
$$

where $C_{1}^{ \pm}$and $C_{2}^{ \pm}$are vectors containing the magnitudes of out and incoming partial waves on planes $S_{1}$ and $S_{2}$, respectively. Incidence of a wave with the 'discretised transverse distribution $s$ on the plane $S_{1}$ may be expressed as

$$
C^{-}=\left[\begin{array}{c}
C_{1}^{-} \\
-C_{2}^{-}
\end{array}\right]=\left[S_{c}\right]\left[\begin{array}{c}
s \\
O O
\end{array}\right]=\left[\begin{array}{cc}
S^{11} & s \\
\hdashline S^{21^{-}} & s
\end{array}\right]=\left[\begin{array}{c}
R s \\
\hdashline T s
\end{array}\right]
$$

where $C_{1}^{+}=s=\left[s_{n}\right]$ on $S_{1}$ and $C_{2}^{+}=\boldsymbol{O}$ on $S_{2}$. The complex magnitudes of the reflected and incident waves differ by the reflectivity $R$ and similarly in the case of the transmitted wave, so

$$
\begin{gathered}
R=\frac{\left(S^{11} s\right)_{n}}{S_{n}} \\
T=\frac{\left(S^{21} s\right)_{n}}{s_{n}}
\end{gathered}
$$

where the index $n$ denotes the $n$th co-ordinate of vector $S^{11} s$.

To determine propagation constants and a transverse mode distribution in a waveguide a transmission matrix $\boldsymbol{A}_{\text {c }}$ must be determined from the scattering one. This matrix relates electric and magnetic fields on two planes separated by a distance $\Delta$

$$
\left[\begin{array}{c}
\boldsymbol{a}_{1} \\
\hdashline \boldsymbol{b}_{1}
\end{array}\right]=\left[A_{c}\right]\left[\begin{array}{c}
\boldsymbol{a}_{2} \\
\hdashline-\boldsymbol{b}_{2} \\
-\boldsymbol{b}_{2}
\end{array}\right]=\left[A_{c}\right]\left[\begin{array}{c}
\boldsymbol{a}_{1} \\
\hdashline \boldsymbol{b}_{1}
\end{array}\right] e^{-j \beta \Delta_{2}}
$$

From this relation the eigenvalue problem equation is obtained:

$$
\left[A_{\mathrm{c}}\right]\left[\begin{array}{c}
\boldsymbol{a}_{1} \\
\bar{b}_{1}
\end{array}\right]=\mathscr{H}\left[\begin{array}{c}
\boldsymbol{a}_{1} \\
\bar{b}_{1}
\end{array}\right] \quad \mathscr{H}=e^{j \beta \Delta_{z}}
$$

The propagation constant $\beta$ is determined from the eigenvalue $\mathscr{H}$ and the transverse mode distribution is given by the eigenvector of the matrix $\boldsymbol{A}_{\mathrm{c}}$.

The convergence behaviour is shown further in the text and in Fig. 7.

The universal code in standard Fortran 77 working on an IBM 3090 computer has been developed on the basis of the technique described. The code evaluates the reflectivity and the transmissivity of an arbitrary obstacle in a rectangular waveguide (linear and isotropic media only) Modifications of the code are applied to the solution of shielded microstrips, the microstrip equivalent homogeneous waveguide with magnetic side walls and electrical top and bottom walls, the E-plane tee junctions in rectangular waveguides, the evaluation of transverse mode distribution and propagation constants in waveguide lines having arbitrary cross-section. There are both 3D and $2 \mathrm{D}$ versions of the code; the $2 \mathrm{D}$ version can run on PCs under MS-DOS. Storage requirements and CPU time depend on the structure complexity (the size of matrices used); here is the main limit to the code application.

IEE PROCEEDINGS-H, Vol. 139, No.4, AUGUST 1992
The code reads the form of analysed volume 'discretisation' and block parameters (dimensions and the complex permitivity). It then calculates the scattering matrices of different block sorts. The main task is to determine the matrix $S_{c}$. This step takes the major part of CPU time. The scattering matrices of blocks are added one by one in rows, row by row, layer by layer to evaluate the resulting matrix. Auxiliary blocks are automatically connected between blocks with different material parameters. They ensure wave reflection between these two blocks. The code for the eigenvalue problem solution (eqn. 13) works with only one layer of blocks having a longitudinal dimension $\Delta_{z}$. This technique must precede the solution of shielded microstrip problems, i.e. one must determine the transverse mode distribution $s$ used in eqns. 10 and 11 in advance.

\section{Discussion of results}

The code presented could be used for the analysis of various structures, as mentioned in the Introduction. Some results are summarised next

\subsection{Structures in rectangular waveguide}

Use of the $2 \mathrm{D}$ code version is easier than the $3 \mathrm{D}$ code. The $2 \mathrm{D}$ version is applicable to structures where the electromagnetic field does not depend on the $y$-co-ordinate (direction parallel to a shorter waveguide side). The computational process is relatively fast, so that complicated structures can be analysed. The analysis of bandpass filters is reported in Section 3.2 as an example.

Results of the analysis of a lossy dielectric cylinder are presented here. This problem was solved using the orthogonal expansion method of the authors of Reference 3. Their data, both theoretical and experimental, are plotted in Fig. 2 in comparison with the data obtained by the MAB technique. Agreement is good. The cylinder cross-section and its vicinity were divided into the net of blocks according Fig. 3. Such a fine net could be used in the $2 \mathrm{D}$ case only because of enormous storage demands and CPU time of the 3D code version. So structures that need the $3 \mathrm{D}$ analysis must be simpler.

An example of a such structure is an E-plane ridge discontinuity. This was analysed in Reference 4 using the generalised scattering matrix method. The results of analysis performed in Reference 4 agree with the experimental data very well and are shown in Fig. 4. Here they are compared with the data obtained by the MAB technique using the $3 \mathrm{D}$ code version. The shape of both curves is similar, shifted on a frequency scale. The agreement could be improved using a finer net of blocks (see Fig. 7).

\subsection{Bandpass filters}

Bandpass filters with inductive shunt obstacles as coupling elements have been analysed by the simple $2 \mathrm{D}$ code version. The solution of a bandpass filter designed for the $96 \mathrm{GHz}$ frequency band (waveguide $\mathrm{R} 740$ ) is shown as a first example. The filter was analysed and measured on a scale model in the $\mathrm{R} 100$ waveguide using scaling parameter $\alpha=0.136$. Fig. 5 shows the transmissivity of the filter together with the structure geometry. Theoretical and experimental data are in agreement.

A more complicated structure was reported in Reference 5: a bilateral filter designed for D-band. Fig. 6 shows data obtained by the code (MAB technique), the results of a measurement and an analysis reported in Reference 5. The inset shows the structure geometry. The 
analysis as a step of an optimisation procedure done in Reference 5 was based on the generalised scattering matrix method. Agreement of the data in Fig. 6 is good.
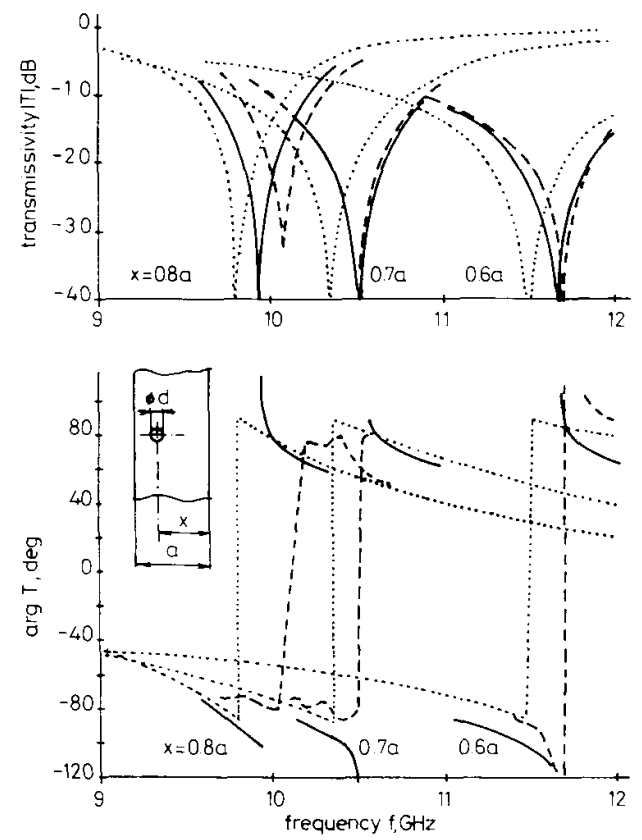

Fig. 2 Transmission of lossy dielectric cylinder in rectangular waveguide $R 100$

$a=22.86 \mathrm{~mm} ; d=1.372 \mathrm{~mm} ; \epsilon_{\mathrm{r}}=38.5 ; \operatorname{tg} \delta=2.10^{-4}$

MAB technique

- - - experiment [3]

theory [3]

Similar filters have been proposed recently for microstrip lines [6]. Inductive obstacles are realised by shunt posts in a dielectric substrate. The filter is designed using calculated characteristics of a single post obtained by the multimode expansion method in an equivalent homogeneous waveguide. The shunt posts analysed subsequently are in fact parts of such a filter.

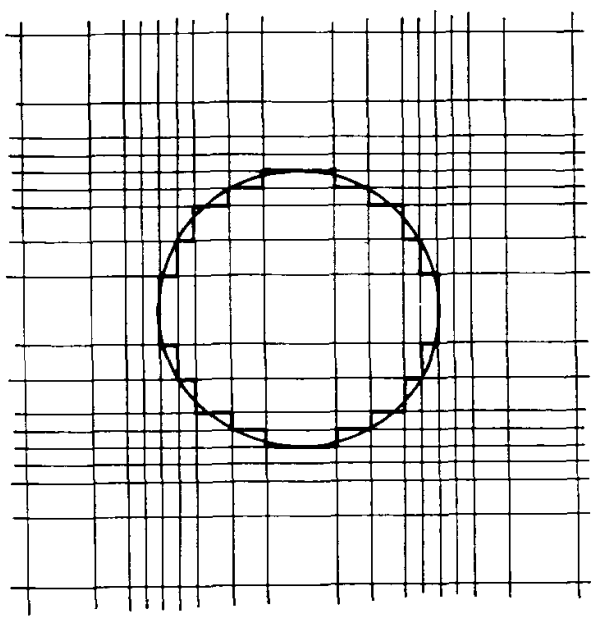

fig. 3 Net of blocks in vicinity of cylinder from Fig. 2(2D problem)
Another type of bandpass filter has been described in Reference 7. The structure of this direct-coupled millimetre-wave bandpass filter is shown in Fig. 7a. The

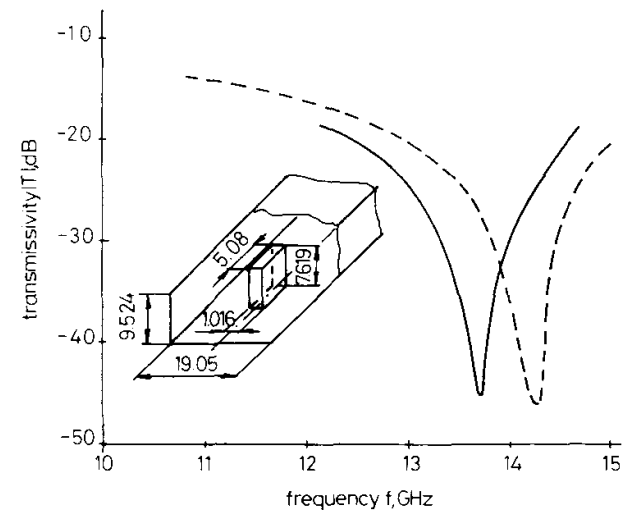

Fig. 4 Transmission of E-plane ridge waveguide discontinuity MAB technique

[4]

filter consists of four resonators coupled by inductive irises. The measured [7] and calculated transmission characteristics are compared in Fig. $7 b$. There are three curves obtained by the $2 \mathrm{D}$ code version in Fig. $7 b$ which describe the convergence of the MAB method. The characteristics were computed using different block dimensions in the vicinity of inductive irises (their edges) $\left(d_{x}=\right.$ $0.118,0.0875,0.05833 \mathrm{~mm}$ ). Dimensions of other blocks in the net were changed proportionately.

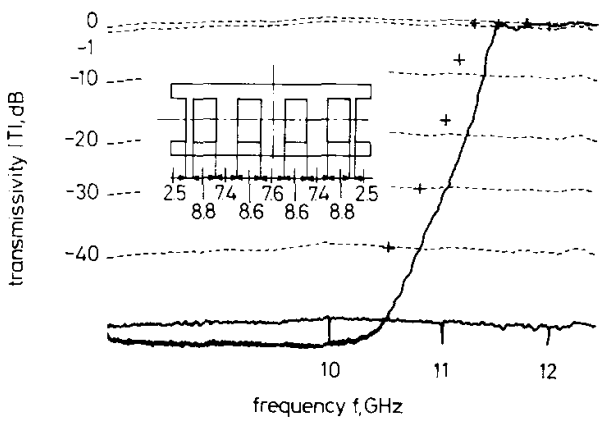

a

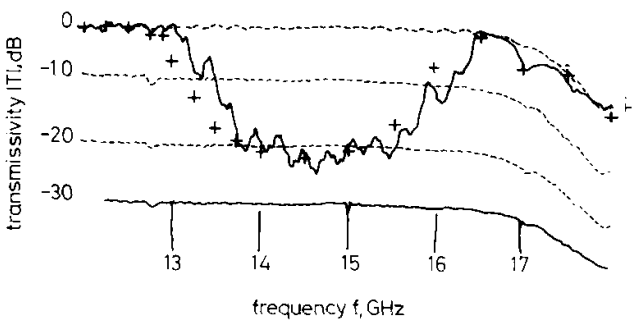

b

Fig. 5 Transmission of bandpass filter a Lower frequency band $b$ Upper frequency band
$+\quad$ MAB technique \begin{tabular}{c} 
MAB techniq \\
$+\quad$ experiment \\
\hline
\end{tabular}

IEE PROCEEDINGS-H, Vol. 139, No.4, AUGUST 1992 
3.3 E-plane Tiunction

Another possible code application is the solution of the E-plane tee junction in a rectangular waveguide. The 3D

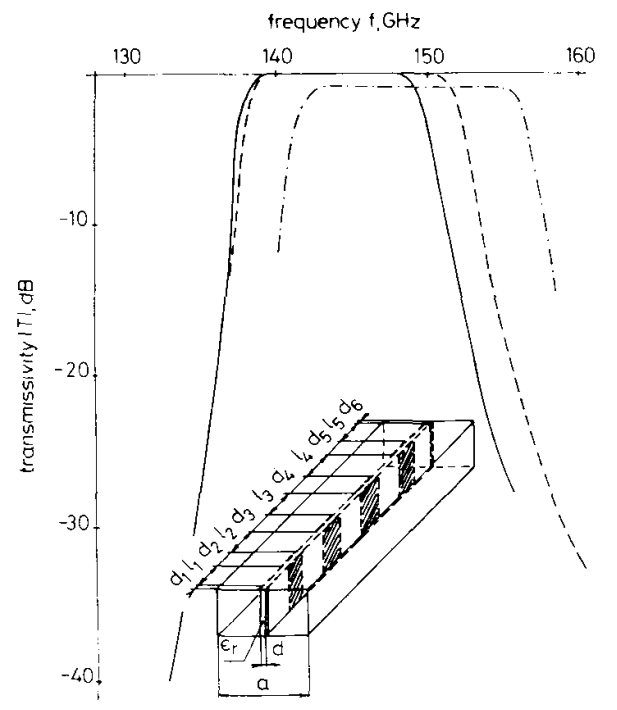

Fig. 6 Results of the bilateral bandpass filter analysis

- MAB technique

-..- theory [s]

- experiment [5]

$a=1.651 \mathrm{~mm} ; \quad d=0.0762 \mathrm{~mm} ; \quad \varepsilon_{0}=2.1, \quad d_{1}=d_{6}=0.03175 \mathrm{~mm}: \quad d_{2}=d_{s}=$ $0.3365 \mathrm{~mm} ; \quad d_{3}=d_{4}=0.4318 \mathrm{~mm} ; \quad l_{1}=l_{5}=0.887 \mathrm{~mm} ; \quad l_{2}=l_{4}=0.8989 \mathrm{~mm}$ $l_{3}=0.8992 \mathrm{~mm}$
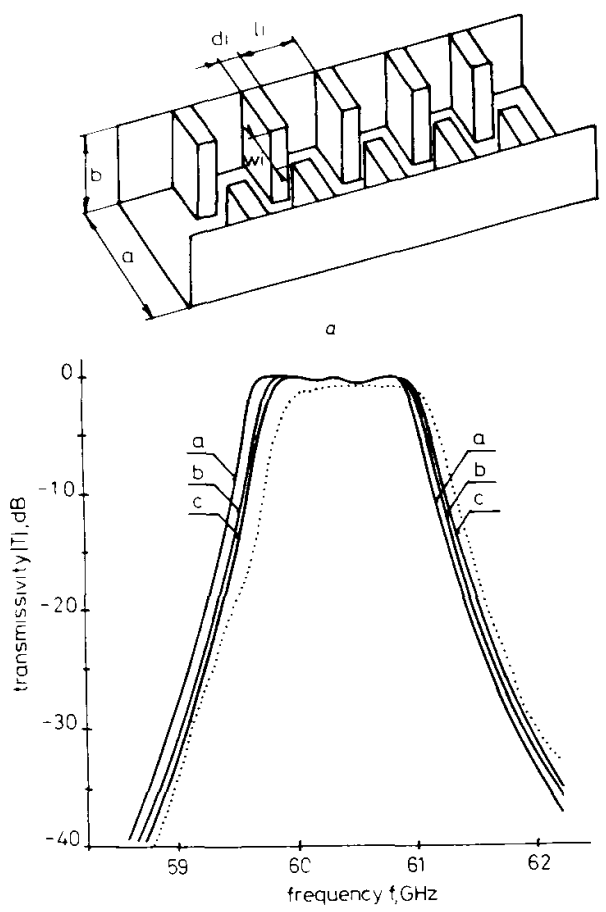

Fig. 7 Structure of the direct-coupled bandpass filter [7] $a=3.7592 \mathrm{~mm} ; b=1.8796 \mathrm{~mm} ; d_{1}=d_{5}=0.56 \mathrm{~mm} ; w_{1}=w_{5}=1.88 \mathrm{~mm} ; d_{2}=$ $d_{4}=0.52 \mathrm{~mm} ; w_{2}=w_{4}=1.18 \mathrm{~mm} ; d_{3}=0.63 \mathrm{~mm} ; w_{3}=1.18 \mathrm{~mm} ; l_{1}=l_{4}=$ $2.73 \mathrm{~mm}: l_{2}-l_{3}=3.02 \mathrm{~mm}$

IEE PROCEEDINGS-H, Vol. 139, No. 4, AUGUST 1992 version of the code was adapted to the computation of a three-port junction scattering matrix. This matrix was computed in dependence on frequency and on branch waveguide height $b_{3}$ variation. All waveguide branches are expected to be loaded without reflections. The calculated and measured data are in a good accordance as it could be seen from Fig. 8. The data plotted here are valid

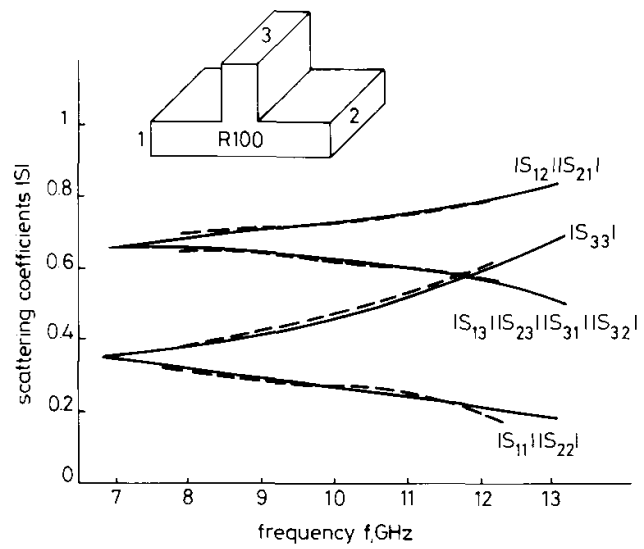

Fig. 8 Scattering matrix elements of E-plane tee junction in rectangular waveguide $R 100$

$b_{1}=b_{2}=b_{3}=10.16 \mathrm{~mm}$

MAB technique

for the waveguide $\mathbf{R} 100$ and the case $b_{3}=b_{1}=b_{2}$. The dependence of the same parameters on the value of $b_{3}$ is shown in Fig. 9 for a frequency of $10 \mathrm{GHz}$. Owing to the symmetry of the E-plane tee junction the following equalities are held: $S_{11}=S_{22}, S_{12}=S_{21}, S_{13}=S_{31}, S_{23}=$ $S_{32},\left|S_{13}\right|=\left|S_{23}\right|, \quad \arg S_{13}=\arg S_{23}-180^{\circ}$. Approximations of these elements by polynomial functions with dependence on frequency and height $b_{3}$ variation are given in Section 6. The approximations were made for the waveguides $R \quad 100$ and $R 320$ in frequency ranges from 8 to $13 \mathrm{GHz}$ and 27.5 to $40 \mathrm{GHz}$, respectively, and in the range of heights from $b_{3}=b_{1}$ to $b_{1} / 2$. A scaling technique can transform these data to frequency bands of other waveguides with the height-to-width ratio $b / a=0.5$.

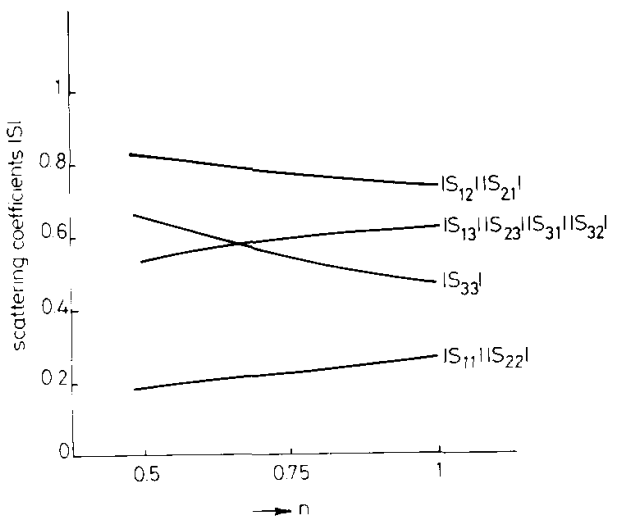

Fig. 9 Dependence of E-plane tee junction scattering matrix elements on relative height of branch waveguide $n\left(b_{3}=n b_{1}\right)$

Waveguide $\mathrm{R} 100 ; \mathrm{f}=10 \mathrm{GHz}$ 
These structures have been analysed recently usually proposing an equivalent circuit (e.g. in Reference 8) Nevertheless, the results in the form of the scattering matrix with its elements approximated by simple functions are more convenient for use in practice.

There is, of course, a possibility to apply the code to the solution of junctions with more complicated structure (e.g. involving filters).

\subsection{Microstrip lines}

Microstrip lines could be analysed using the code in two ways. The choice depends on the complexity of the structure to be solved. The simple way is to use an equivalent homogeneous waveguide with effective parameters $W_{\text {efr }}$ and $\varepsilon_{\text {r.eff }}$ that has top and bottom walls perfectly conducting and magnetic side walls. The second way is to solve a microstrip as a shielded microstrip line. This last case requires the $3 \mathrm{D}$ code version whereas the first is solved as a $2 \mathrm{D}$ problem.

The single shunt post in a microstrip line was solved using both methods. Results are plotted in Fig. 10, where the structure geometry is shown in the inset. The calculated data are compared with the result of an experiment by W. Menzel.

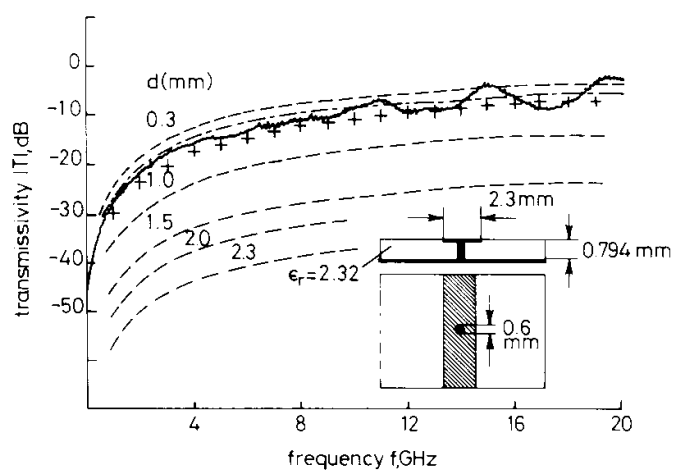

Fig. 10 Analysis of shunt post in microstrip line experiment [8] $+\quad 2 \mathrm{D}$ analysis using an equivalent homogeneous waveguide post diameter $\phi=0.6 \mathrm{~mm}$

her post diameters

JD analysis of shielded microstrip line

The agreement is good. The other data plotted in Fig 10 were calculated using an equivalent homogeneous waveguide for structures with different post diameters.

The computational procedure in the case of a shielded microstrip line is not so simple as in a rectangular waveguide where the distribution of the incident mode is known.

In an equivalent homogeneous waveguide the distribution of a propagating mode is constant within the whole cross-section. Solving the task in a shielded microstrip line must use the code for the evaluation of a propagating fundamental mode distribution. Its results serve as the input data for the 3D code.

The results of both techniques differ slightly from the experimental data. A homogeneous equivalent waveguide is a simplified tool for the solution of microstrip lines as it does not take into consideration the proper distribution of a fringing field. On the other hand, the 2D code can use a much finer net of blocks than in the 3D analysis of a shielded microstrip line.

356

\section{Conclusions}

The MAB technique is the basis on which the universal code was developed. Various structures used in microwave and millimetre-wave technique have been analysed by this code: structures in a rectangular waveguide, bandpass filters with shunt inductive obstacles, E-plane tee junction in a rectangular waveguide, and the discontinuities in microstrip lines, were solved by means of an equivalent homogeneous waveguide and a shielded microstrip

There are two basic versions of the code. The simple version solves $2 \mathrm{D}$ tasks where the electric field is parallel to the shorter side of a waveguide and is uniform in this direction (solution of the shunt obstacles etc.). This version is relatively fast and can be used for complex structures. It gives results in good agreement with the experimental data. The application of the $3 \mathrm{D}$ version is not so easy and is limited to simple structures. The problem is not in the method or in the code but in the enormous storage demands and CPU time. In spite of this, the code seems to be a very effective tool for the analysis of a wide variety of structures.

A detailed analysis of the E-plane tee junction in the rectangular waveguide $R 100$ and $R 320$ was performed. The scattering matrix of this three-port junction was calculated in dependence on frequency and on the branch waveguide height variation. Results were approximated by polynomial functions convenient for general use.

\section{References}

I MACHÁC, J.: 'Inhomogenity analysis of waveguides with $\mathrm{TE}_{10}$ dominant mode', Slaboproudý obzor, 1990, 51, (6), pp. 235-240 (in Czech)

2 NIKOLSKIJ, VY, and NIKOLSKAJA, II. 'Decomposition technique in tasks of electrodynamics' (Nauka, Moscow, 1983) (in Russian)

3 GESCHE, R., and LÖCHEL, N.: 'Scattering by a lossy dielectric cylinder in a rectangular waveguide', IEEE Trans., 1988, MTT-36, (1), pp. 137-144

4 MANSOUR, R.R., TONG, R.S.K., and MACPHIE, R.H.: 'Simplifed description of the field distribution in finlines and ridge waveguides and its application to the analysis of E-plane discontinuities', IEEE Trans 1988, MTT-36, (12), p. 1825

Trans., 1988, MTT-36, (12), p. 1825
5 BUI, L.Q., BALL, D., and IHOH, T.: 'Broadband millimeter-wave BUI, L.Q., BALL, D., and IHOH, T.: 'Broadband millimeter-wave
E-plane bandpass filters', IEEE Trans., 1984, MTT-32, (12), pp. $1655-1658$

6 FINCH, K.L., and ALEXOPOULOS, N.G.: 'Shunt posts in microstrip transmission lines', IEEE Trans., 1990, MTT-38, (11), pp. 15851594

7 KLAASSEN, A BARTH, $\mathrm{H}$., and MENZEL, W.: 'Effeckiver Entwurt und Aufbau von mm-Wellen-Hoblleiter-Bandpassfiltern' Entwurt und Aufbau von mm-Wellen-Hohlleiter-Bandpassfiltern. April 1990, pp. 534-536

8 OBATA, T., and CHIBA, J.: 'Improved theory for E-plane symmetrical tee junctions', IEEE Trans., 1989, MTT-37, pp. 624-627

\section{$6 \quad$ Appendix}

The scattering matrix of the E-plane tee junction in the $R$ 100 and $R 320$ waveguides was evaluated using frequency and branch waveguide height $b_{3}$ as variables. Frequency varies from 8 to $13 \mathrm{GHz}$ for the $R 100$ waveguide and from 27.5 to $40 \mathrm{GHz}$ for the $\mathbf{R} 320$ waveguide. The height $b_{3}$ varies from $b_{1}$ to $b_{1} / 2$ in the both cases. Relative height value $n$ is used via the relation $b_{3}=n b_{1}$, where $b_{1}$ is the straight waveguide height, so $n$ varies from 0.5 to 1 .

IEE PROCEEDINGS-H, Vol. 139, No. 4, AUGUST 1992 
Table 1 : Approximation for $R 100$ waveguide E-plane tee junction

\begin{tabular}{lcccccccccc}
\hline & \multicolumn{9}{c}{$a_{0}$} & \multicolumn{9}{c}{$a_{1}$} \\
& \cline { 2 - 10 } & $b_{00}$ & $b_{01}$ & $b_{02}$ & $b_{10}$ & $b_{11}$ & $b_{12}$ & $b_{20}$ & $b_{21}$ & $b_{22}$ \\
\hline$\left|S_{11}\right|$ & -0.6251 & 2.0393 & -0.7769 & 0.1353 & -0.3278 & 0.1456 & -0.00666 & 0.01527 & 0.00764 \\
$\arg S_{11}$ & 303.2 & -936.1 & 578.1 & -62.97 & 177.6 & -107.5 & 2.123 & -7.547 & 4.107 \\
$\left|S_{12}\right|$ & 1.0377 & -0.442 & -0.04532 & -0.01167 & 0.004 & 0.02347 & 0.00044 & 0.00133 & -0.00071 \\
$\arg S_{12}$ & 48.87 & 126.9 & -24.27 & -10.79 & -27.8 & 5.92 & 0.2633 & 0.7333 & -0.3733 \\
$\left|S_{13}\right|$ & 0.239 & 0.5873 & -0.3227 & 0.0197 & 0.0104 & 0.0176 & -0.0009 & -0.00213 & -0.00053 \\
$\arg S_{13}$ & 211.9 & 91.73 & -43.2 & -8.72 & -17.38 & 9.2 & 0.0733 & 0.3267 & -0.4 \\
$\left|S_{33}\right|$ & 0.854 & 0.00132 & -0.592 & 0.0284 & -0.1916 & 0.1472 & -0.0018 & 0.0119 & -0.0064 \\
$\arg S_{33}$ & 40.23 & -393.3 & 361.3 & -2.76 & 49.86 & -47.28 & -0.6133 & -1.7533 & 1.627 \\
\hline
\end{tabular}

Table 2: Approximation for $\mathbf{R} 320$ waveguide E-plane tee junction

\begin{tabular}{|c|c|c|c|c|c|c|c|c|c|}
\hline & \multicolumn{3}{|c|}{$a_{0}$} & \multicolumn{3}{|c|}{$a_{1}$} & \multicolumn{3}{|c|}{$a_{2}$} \\
\hline & $b_{\infty 0}$ & $b_{01}$ & $b_{02}$ & $b_{10}$ & $b_{11}$ & $b_{12}$ & $b_{20}$ & $b_{21}$ & $b_{22}$ \\
\hline $\begin{array}{l}\left|S_{11}\right| \\
\arg S_{11} \\
\left|S_{12}\right| \\
\arg S_{12} \\
\left|S_{13}\right| \\
\arg S_{13} \\
\left|S_{33}\right| \\
\arg S_{33}\end{array}$ & $\begin{array}{c}0.1306 \\
863.9 \\
0.8977 \\
43.39 \\
0.2871 \\
234.9 \\
0.4488 \\
-67.82\end{array}$ & $\begin{array}{c}0.5369 \\
-2354.8 \\
-0.0451 \\
157.4 \\
0.1228 \\
9.035 \\
1.837 \\
375.7\end{array}$ & $\begin{array}{c}0.0507 \\
1508.2 \\
-0.2752 \\
-36.59 \\
-0.2 \\
-16.85 \\
-2.0 \\
-211.3\end{array}$ & $\begin{array}{c}-0.00312 \\
-62.48 \\
0.00772 \\
-3.044 \\
0.00284 \\
-4.024 \\
0.03824 \\
4.896\end{array}$ & $\begin{array}{c}-0.00232 \\
160.4 \\
-0.0343 \\
-10.82 \\
0.03344 \\
-0.3677 \\
-0.1855 \\
-26.42\end{array}$ & $\begin{array}{c}-0.0112 \\
-100.9 \\
0.0246 \\
2.944 \\
-8.9 \times 10^{-7} \\
1.344 \\
0.1366 \\
17.09\end{array}$ & $\begin{array}{c}0.000032 \\
1.028 \\
-0.00017 \\
0.0197 \\
-0.000037 \\
0.0224 \\
-0.0007 \\
-0.1483\end{array}$ & $\begin{array}{c}-0.00018 \\
-2.749 \\
0.00078 \\
0.1003 \\
-0.0007 \\
-0.0512 \\
0.00334 \\
0.4096\end{array}$ & $\begin{array}{l}0.00021 \\
1.668 \\
-0.00038 \\
-0.05974 \\
1.5 \times 10^{-8} \\
-0.0171 \\
-0.0021 \\
-0.2901\end{array}$ \\
\hline
\end{tabular}

The resulting values of scattering matrix elements $S_{11}$, $S_{12}, S_{13}$ and $S_{33}$ were approximated by a proper continuous function of two variables. First by a polynomial function of frequency $f$

$$
S_{k l}=a_{0}+a_{1} f+a_{2} f^{2}
$$

where subscripts $k l$ denote $11,12,13$ and 33 . Frequency is substituted in $\mathrm{GHz}$. Each parameter $a_{i}, i=0,1,2$, in eqn. 14 was fitted further by a polynomial function of the relative height $n$

$$
a_{i}=b_{i 0}+b_{i 1} n+b_{i 2} n^{2} \quad i=0,1,2
$$

So as a result

$$
\begin{aligned}
S_{k l}= & b_{00}+b_{01} n+b_{02} n^{2}+\left(b_{10}+b_{11} n+b_{12} n^{2}\right) f \\
& +\left(b_{20}+b_{21} n+b_{22} n^{2}\right) f^{2}
\end{aligned}
$$

Similar relations could be written for arg $S_{k l}$ (units are degrees). The resulting nine parameters $b_{i j}, i, j=0,1,2$, are listed in Table 1 (waveguide $R 100$ ) and in Table 2 (waveguide $\mathrm{R} 320$ ). The frequency and the value $n$ intervals, where the approximation is valid, are given above.

The maximum relative difference with the calculated data is $1.5 \%$ for $n=1$ rising to $4.5 \%$ for $n=0.5$ in the whole frequency range for all elements $S_{k l}$. 